\title{
LEVANTAMENTO FLORÍSTICO E FITOSSOCIOLÓGICO EM DUAS ÁREAS DE CERRADO SENSU STRICTO NO PARQUE ESTADUAL DA SERRA DE CALDAS NO- VAS, GOIÁS
}

\author{
Lucivânio Oliveira Silva ${ }^{2,3}$ \\ Diogo Andrade Costa ${ }^{4}$ \\ Kleber do Espírito Santo Filho ${ }^{4}$ \\ Heleno Dias Ferreira ${ }^{1}$ \\ Divino Brandão ${ }^{1}$
}

Recebido em 20/11/00. Aceito em 25/07/01.

\begin{abstract}
RESUMO - (Levantamento florístico e fitossociológico em duas áreas de cerrado sensu stricto no Parque Estadual da Serra de Caldas Novas, Goiás) A necessidade de se conhecer mais sobre o Bioma Cerrado torna-se cada vez mais urgente, devido à destruição acelerada deste bioma. Este trabalho teve como objetivo realizar um levantamento florístico e fitossociológico em duas áreas de Cerrado sensu stricto, no Parque Estadual da Serra de Caldas Novas, registrando as espécies arbóreas e arbustivas, utilizando o método de Point Centered Quarter. Verificou-se que apesar de algumas diferenças litológicas, latossolo vermelho-escuro na primeira área e vermelho-amarelo na segunda, a similaridade entre elas foi alta, com índices de Jaccard $(0,72)$ e Morisita $(0,64)$. Das 67 espécies pertencentes a 51 gêneros e 29 famílias, 48 foram comuns às duas áreas. Kielmeyera coriacea, Qualea grandiflora, Caryocar brasiliense, Syagrus flexuosa e Ouratea hexasperma tiveram os maiores Índices de Valor de Importância na primeira área e Pouteria ramiflora, Qualea parviflora, Qualea grandiflora, Caryocar brasiliense e Vochysia cinamommea, foram as espécies de maior importância na segunda área. A família Vochysiaceae foi a de maior IVI em ambas as áreas e Leguminosae apresentou o maior número de espécies (15), seguindo Vochysiaceae (7), Apocynaceae (5) e Myrtaceae (4).
\end{abstract}

Palavras-chave - florística, fitossociologia, cerrado, Parque Estadual da Serra de Caldas Novas

ABSTRACT - (Floristic and phytosociology inventory in two areas of "Cerrado" stricto sensu in the Parque Estadual da Serra de Caldas Novas, Goiás). The need to know more about "Bioma Cerrado" becomes more and more urgent, due to the accelerated destruction of this "Biome". The objective of this work was to conduct a floristic inventory in two areas of Cerrado " stricto sensu ", in the Parque Estadual da Serra de Caldas Novas. Arboreal and shruby species were registered, using the Point Centered Quarter Method. It was verified that in spite of some litologic differences, dark red latossoil in the first area and red yellow latossoil in the second one, the two areas showed high similarity, showed by Jaccard's index $(0,72)$ and Morisita's index $(0,64) .67$ species belonging to 51 genera and 29 families, were identified, 48 species common to both areas. Kielmeyera coriacea,

\footnotetext{
' Docentes do Departamento de Biologia Geral, Instituto de Ciências Biológicas, Universidade Federal de Góias. Cx. P. 131. 74001-970, Goiânia, GO, Brasil.

22E-mail: lucivani@hotmail.com

${ }^{3}$ Docente da Associação Educativa Evangélica (AEE) e Universidade Estadual de Góias (UEG).

${ }^{4}$ Discentes de Biologia da UFG.
} 
Qualea grandiflora, Caryocar brasiliense, Syagrus flexuosa and Ouratea hexasperma had larger indexes of importance in the first area and Pouteria ramiflora, Qualea parviflora, Qualea grandiflora, Caryocar brasiliense and Vochysia cinamommea, were the most important species in the second area. The most important family in both areas was Vochysiaceae and Leguminosae was the family that showed the biggest number of species (15), followed by Vochysiaceae (7), Apocynaceae (5) and Myrtaceae (4).

Key words - Floristic, phytosociology, “cerrado”, Parque Estadual da Serra de Caldas Novas

\section{Introdução}

Grande parte das áreas de cerrado já não possui mais a cobertura vegetal original, sendo atualmente ocupada por paisagens antrópicas. Mitermeier et al. (1999) estimaram que 67\% das áreas de Cerrado são consideradas como "altamente modificadas" e apenas $20 \%$ encontram-se em seu estado original. Mesmo as áreas ainda cobertas de paisagem natural, sofrem conseqüentemente os efeitos da poluição dos recursos hídricos, agrotóxicos, erosão, assoreamento, plantas e animais invasores, extrativismo vegetal e animal predatórios, fatores estes decorrentes da industrialização desenfreada e da falta de consciência preservacionista (Reatto et al., 1998).

Segundo Eiten (1993) a flora do cerrado é composta de dois grupos de espécies: árvores e arbustos de caules grossos e a camada rasteira, constituindo aproximadamente 300450 espécies vasculares por hectare, perdendo apenas para a floresta tropical úmida. Fisionomicamente, o Cerrado é constituído de um grande mosaico, que inclui formações florestais com dossel mais ou menos fechado (cerradão), contendo árvores de $12 \mathrm{~m}$ de altura ou mais; cerrado sensu stricto, com um estrato arbóreo-arbustivo geralmente em torno de 6 ou 7 metros e um estrato rasteiro mais ou menos contínuo; campo cerrado apresentando uma vegetação com o estrato arbóreoarbustivo mais aberto; campo sujo, com estrato herbáceo-graminoso dominante e arbustos ou pequenas árvores esparsos; campo limpo, com um único estrato, dominado por gramíneas.
Autores como Goodland (1970); Ratter et al. (1973); Rizzo (1981); Silberbauer-Gottsberger \& Eiten (1983); Ribeiro et al. (1985); Oliveira-Filho \& Martins (1986), Ratter (1986); Nascimento \& Saddi (1992); Ratter \& Dargie (1992); Felfili et al. (1993); Felfili \& Silva Jr. (1992, 1993); Filgueiras \& Pereira (1993); Mantovani \& Martins (1993); Castro (1994); Felfili (1994); Meirelles \& Barreto Luiz (1995); Ratter et al. (1996), Pedralli et al. (1997) e Almeida et al. (1998) têm feito levantamentos florísticos e fitossociológicos em áreas do cerrado de diferentes regiões do país.

Ratter \& Dargie (1992) e Ratter et al. (1996) compararam diversos trabalhos publicados sobre a vegetação do Cerrado s.s., registrando as espécies arbóreas mais características. Uma das conclusões desses autores é a de que a flora do cerrado apresenta uma grande diversidade devido às variações climáticas e dos tipos de solo.

$\mathrm{O}$ estudo fitossociológico fornece informações sobre a estrutura da comunidade de uma determinada área, além de possíveis afinidades entre espécies ou grupos de espécies, acrescentando dados quantitativos a respeito da estrutura da vegetação. Buscando ampliar as informações sobre vegetação nativa lenhosa de Cerrado, fezse um levantamento florístico e fitossociológico de duas áreas de cerrado sensu stricto no Parque Estadual da Serra de Caldas Novas, verificando as populações arbóreas e arbustivas de maior importância e analisando fatores como densidade da comunidade, distribuição de altura das plantas e variação da circunferência dos caules. 


\section{Materiais e Métodos}

Os dados foram coletados no Parque Estadual da Serra de Caldas Novas (PESCAN), localizado entre os municípios de Caldas Novas e Rio Quente, no sudeste do Estado de Goiás, a 180 km da capital, Goiânia (Almeida \& Sarmento, s.d.). As coletas ocorreram na primeira quinzena do mês de Julho de 1999.

Duas áreas de $300 \mathrm{~m}$ X $200 \mathrm{~m}$ cada foram delimitadas no platô da serra, sendo que a primeira dista cerca de $5 \mathrm{~km}$ da sede do Parque e apresenta um latossolo vermelho-escuro e a segunda fica a $10 \mathrm{~km}$ da sede e apresenta uma variação quanto ao tipo de latossolo, algumas vezes vermelho-escuro e outras vezes, vermelho-amarelo.

Utilizou-se o método do "Point Centered Quarter" (Müeller-Dombois \& Elemberg, 1974), sendo que em cada área foram demarcados 150 pontos distribuídos em 15 linhas, distando 20 $\mathrm{m}$ entre linhas e entre pontos. Foram incluídas na amostra plantas que apresentassem circunferência de caule igual ou superior a $13 \mathrm{~cm}$ no nível do solo, pois os resultados obtidos serviriam para relacionar as espécies arbóreas escolhidas por uma espécie de cupim para construir seus ninhos, sendo que todas as árvores ocupadas pelo inseto apresentavam circunferência acima de $13 \mathrm{~cm}$.

Desta forma, foram incluídas 600 plantas por área, sendo que, de cada uma registrou-se: nome científico, altura total da planta, circunferência do caule ao nível do solo e distância ao ponto. A maioria das plantas foi identificada in loco e as demais foram herborizadas e posteriormente identificadas por comparação com o material depositado no Herbário da Universidade Federal de Goiás (UFG). Para cada espécie vegetal, foram calculados os valores absolutos e relativos de densidade, freqüência e dominância. Com o somatório dos valores relativos, obteve-se o índice de valor de importância (IVI).
A similaridade florística entre as áreas foi calculada através do coeficiente de Jaccard e do Índice de Morisita (Krebs, 1989). O coeficiente de Jaccard serviu também para medir a similaridade entre as duas áreas do PESCAN e outras áreas estudadas por vários autores. Aplicou-se o Teste t (Sokal \& Rohlf, 1995) entre as medidas de altura total das plantas e a circunferência dos caules das duas áreas estudadas.

\section{Resultados e Discussão}

Foram registradas 67 espécies, pertencentes a 51 gêneros e 29 famílias, sendo 56 espécies na área 1 e 59 na área 2. Destas, 8 espécies apareceram apenas na área 1 e 11 foram encontradas somente na área 2, totalizando assim, 48 espécies comuns. A relação das espécies por família encontra-se na Tab. 1. A família Leguminosae foi a que apresentou o maior número de espécies (15), seguida de Vochysiaceae (7), Apocynaceae (5) e Myrtaceae (4).

Leguminosae tem sido a família mais diversificada na maioria dos levantamentos realizados no cerrado (Ribeiro et al., 1985; Oliveira Filho \& Martins, 1986; Nascimento \& Saddi, 1992; Filgueiras \& Pereira, 1993 e Mantovani \& Martins, 1993) embora outras famílias também já tenham sido citadas nesta posição como Rubiaceae e Myrtaceae. Segundo Oliveira-Filho et al. (1989), dependendo das condições do meio, determinada espécie será melhor adaptada a uma área que outra.

A família Vochysiaceae apresentou maior IVI em ambas as áreas, sendo verificado o mesmo resultado em Planaltina - DF (Ribeiro et al., 1985) e em uma área de cerrado da Salgadeira MT (Oliveira Filho \& Martins, 1986). Qualea grandiflora apresentou-se com IVI alto nas áreas 1 e 2 , ocupando a $2^{\mathrm{a}}$ a $3^{\mathrm{a}}$ posições respectivamente (Tab. 2 e 3). Ratter \& Dargie (1992) e Ratter et al. (1996) ao analisarem vários trabalhos de levantamento florístico em áreas de cerrado observaram que ela foi a espécie mais 
Tabela 1. Relação de espécies arbóreas e arbustivas em duas áreas analisadas no Parque Estadual da Serra de Caldas Novas, com as suas respectivas famílias.

\begin{tabular}{|c|c|}
\hline FAMÍLIA & ESPÉCIE \\
\hline ANNONACEAE & Annona crassiflora Mart. \\
\hline \multirow[t]{5}{*}{ APOCYNACEAE } & Aspidosperma dasicarpon A DC. \\
\hline & Aspidosperma macrocarpon A DC. \\
\hline & Aspidosperma tomentosum A DC \\
\hline & Hancornia speciosa Gomez \\
\hline & Himatanthus obovatus (M. Arg.)R.E. Woodson \\
\hline ARALIACEAE & Schefflera macrocarpon (Serm.) D.C. Froolik \\
\hline BIGNONIACEAE & Tabebuia aurea (Mart.) Bur.Tabebuia ochracea (Cham.) Standl. \\
\hline CARYOCARACEAE & Caryocar brasiliense Camb. \\
\hline CELASTRACEAE & Austroplenckia populnea (Reiss) Lund. \\
\hline CHRYSOBALANACEAE & Licania humilis Cham \& Schlect \\
\hline \multirow[t]{2}{*}{ COMPOSITAE } & Eremanthus sp. \\
\hline & Piptocarpha rotundifolia (Less.) Baker \\
\hline \multirow[t]{3}{*}{ CONNARACEAE } & Connarus sp. \\
\hline & Connarus suberosus Planch. \\
\hline & Rourea induta Planch. \\
\hline EBENACEAE & Diospyros hispida A DC. var camporum Warm. \\
\hline \multirow[t]{2}{*}{ ERYTHROXYLACEAE } & Erythroxylum suberosus St. Hil. \\
\hline & Erythrocylum tortuosum Mart. \\
\hline FLACOURTIACEAE & Casearia sylvestris $\mathrm{Sw}$ \\
\hline \multirow[t]{2}{*}{ GUTTIFERAE } & Kielmeyera coriacea Mart. \\
\hline & Kielmeyera speciosa St. Hil. \\
\hline LABIATAE & Hyptidendron canum (Pohl. ex. Benth) RM. Harley \\
\hline \multicolumn{2}{|l|}{ LEGUMINOSAE } \\
\hline \multirow[t]{5}{*}{ CAESALPINIOIDEAE } & Acosmium dasycarpum (Vog.) Yakovl. \\
\hline & Hymenea stigonocarpa Mart. ex. Hayne \\
\hline & Peltogyne sp. \\
\hline & Sclerolobium aureum (Tul.) Benth. \\
\hline & Sclerolobium paniculatum Vog. \\
\hline \multirow[t]{4}{*}{ MIMOSOIDEAE } & Enterolobium gummiferum (Mart.) Macb. \\
\hline & Platymenia reticulata Benth. \\
\hline & Stryphnodendron adstringens (Mart.) Coville \\
\hline & Stryphnodendron polyphyllum Mart. var. villosum \\
\hline \multirow[t]{6}{*}{ PAPILIONIOIDEAE } & Andira sp. \\
\hline & Bowdichia virgiloides $\mathrm{H}$. В. \& K \\
\hline & Dalbergia miscolobium Benth \\
\hline & Machaerium sp. \\
\hline & Pterodon emaginatus Vog. \\
\hline & Vatairea sp. \\
\hline LOGANIACEAE & Sthrychnos pseudo-quina St. Hil. \\
\hline LYTHRACEAE & Lafoensia pacari St. Hil. \\
\hline \multirow[t]{2}{*}{ MALPIGHIACEAE } & Byrsonima coccolobifolia $\mathrm{HB} \& \mathrm{~K}$. \\
\hline & Byrsonima verbascifolia Rich. ex. Juss. \\
\hline \multirow[t]{2}{*}{ MELASTOMATACEAE } & Leandra $\mathrm{sp}$. \\
\hline & Miconia sp. \\
\hline MORACEAE & Brosimum gaudichaudii Tréc. \\
\hline MYRSINACEAE & Myrsine guianensis (Aubl.) Kuntz \\
\hline \multirow[t]{4}{*}{ MYRTACEAE } & Myrcia sp. \\
\hline & Myrcia lingua Berg. \\
\hline & Psidium australe Camb. \\
\hline & Psidium sp. \\
\hline NYCTAGINACEAE & Neea theifera Oerst. \\
\hline OCHNACEAE & Ouratea hexasperma (St. Hil.) Benth. \\
\hline PALMAE & Syagrus flexuosa (Mart.) Becc. \\
\hline PROTEACEAE & Roupala montana Aubl. \\
\hline \multirow[t]{2}{*}{ RUBIACEAE } & Palicourea rigida Kunth. \\
\hline & Tocoyena formosa (C \& S.) Schum \\
\hline SAPOTACEAE & Pouteria ramiflora (Mart.) Radlk \\
\hline STYRACACEAE & Styrax ferrugineus Nees \& Mart. \\
\hline \multirow{7}{*}{ VOCHYSIACEAE } & Qualea grandiflora Mart. \\
\hline & Qualea multiflora Mart. \\
\hline & Qualea parviflora Mart. \\
\hline & Salvertia convallarieodora St. Hil \\
\hline & Vochysia cinnamomea Pohl. \\
\hline & Vochysia tomentosum Voch. \\
\hline & Vochysia tucanorum Mart. \\
\hline
\end{tabular}


Tabela 2. Espécies e parâmetros fitossociológicos, ordenados de acordo com os valores do Índice IVI (Índice de Valor de Importância) das espécies na área 1. Parque Estadual da Serra de Caldas Novas (1999).

\begin{tabular}{|c|c|c|c|c|c|c|c|c|c|}
\hline Seq. & Espécies vegetais da Área 1 & $\mathbf{N}$ & $\mathbf{D A}_{\mathbf{x}}$ & $\mathbf{F A}$ & $\operatorname{DoA}_{x}$ & $\mathbf{D R}_{\mathrm{x}}$ & $\mathbf{F R}$ & $\operatorname{DoR}_{x}$ & IVI \\
\hline 1 & Kielmeyera coriacea Mart. & 66 & 209,77 & 42,00 & 1,738 & 11,00 & 11,86 & 10,69 & 33,55 \\
\hline 2 & Qualea grandiflora Mart. & 42 & 133,49 & 22,66 & 1,943 & 7,00 & 6,40 & 11,95 & 25,36 \\
\hline 3 & Caryocar brasiliense Camb. & 30 & 95,35 & 18,00 & 2,015 & 5,00 & 5,08 & 12,39 & 22,48 \\
\hline 4 & Syagrus flexuosa (Mart.) Becc. & 41 & 130,31 & 23,33 & 0,729 & 6,83 & 6,59 & 4,48 & 17,91 \\
\hline 5 & Ouratea hexasperma (St. Hil.) Baill. & 35 & 111,24 & 18,66 & 1,106 & 5,83 & 5,27 & 6,80 & 17,91 \\
\hline 6 & Qualea multiflora Mart. & 29 & 92,17 & 16,66 & 0,833 & 4,83 & 4,70 & 5,12 & 14,66 \\
\hline 7 & Qualea parviflora Mart. & 32 & 101,70 & 17,33 & 0,583 & 5,33 & 4,89 & 3,59 & 13,82 \\
\hline 8 & Annona crassiflora Mart. & 22 & 69,92 & 12,66 & 0,944 & 3,66 & 3,57 & 5,80 & 13,05 \\
\hline 9 & Eremantus sp. & 31 & 98,52 & 14,66 & 0,320 & 5,16 & 4,14 & 1,96 & 11,27 \\
\hline 10 & Byrsonima verbascifolia Rich. ex. Juss. & 21 & 66,74 & 13,33 & 0,461 & 3,50 & 3,76 & 2,83 & 10,10 \\
\hline 11 & Stryphnodendron adstringens (Mart.) Coville & 20 & 63,56 & 11,33 & 0,475 & 3,33 & 3,20 & 2,92 & 9,45 \\
\hline 12 & Hancornia speciosa Gomez & 19 & 60,38 & 12,00 & 0,285 & 3,16 & 3,38 & 1,75 & 8,31 \\
\hline 13 & Psidium $\mathrm{sp}$. & 19 & 60,38 & 12,00 & 0,280 & 3,16 & 3,38 & 1,72 & 8,28 \\
\hline 14 & Piptocarpha rotundifolia (Less.) Baker & 16 & 50,85 & 9,33 & 0,409 & 2,66 & 2,63 & 2,52 & 7,82 \\
\hline 15 & Stryphnodendron polyphyllum Mart. var. villosum 1 & 17 & 54,03 & 10,66 & 0,147 & 2,83 & 3,01 & 0,90 & 6,75 \\
\hline 16 & Aspidosperma dasicarpon A .DC. & 17 & 54,03 & 8,66 & 0,220 & 2,83 & 2,44 & 1,35 & 6,63 \\
\hline 17 & Dalbergia miscolobium Benth. & 6 & 19,07 & 4,00 & 0,664 & 1,00 & 1,12 & 4,08 & 6,20 \\
\hline 18 & Erythroxylum suberosus St. Hil. & 15 & 47,67 & 8,00 & 0,145 & 2,50 & 2,25 & 0,89 & 5,65 \\
\hline 19 & Byrsonima coccolobifolia H.B. \& K. & 10 & 31,78 & 6,66 & 0,189 & 1,66 & 1,88 & 1,16 & 4,71 \\
\hline 20 & Licania humilis Cham \& Schlect. & 8 & 25,42 & 5,33 & 0,262 & 1,33 & 1,50 & 1,61 & 4,45 \\
\hline 21 & Pterodon emaginatus Vog. & 4 & 12,71 & 1,33 & 0,508 & 0,66 & 0,37 & 3,12 & 4,17 \\
\hline 22 & Palicourea rigida Kunth. & 7 & 22,24 & 4,66 & 0,067 & 1,16 & 1,31 & 0,41 & 2,89 \\
\hline 23 & Brosimum gaudichaudii Tréc. & 7 & 22,24 & 4,66 & 0,059 & 1,16 & 1,31 & 0,36 & 2,85 \\
\hline 24 & Styrax ferrugineus Nees \& Mart. & 6 & 19,07 & 4,00 & 0,116 & 1,00 & 1,12 & 0,71 & 2,84 \\
\hline 25 & Aspidosperma macrocarpon A .DC & 6 & 19,07 & 4,00 & 0,112 & 1,00 & 1,12 & 0,69 & 2,82 \\
\hline 26 & Diopyros hispida A .DC var. camporum Warm. & 7 & 22,24 & 4,66 & 0,054 & 1,16 & 1,31 & 0,33 & 2,81 \\
\hline 27 & Lafoensia pacari St. Hil. & 3 & 9,53 & 2,00 & 0,224 & 0,50 & 0,56 & 1,38 & 2,44 \\
\hline 28 & Strychnos pseudo-quina St. Hil. & 4 & 12,71 & 2,66 & 0,166 & 0,66 & 0,75 & 1,02 & 2,44 \\
\hline 29 & Tocoyena formosa K. Schum. & 5 & 15,89 & 2,66 & 0,031 & 0,83 & 0,75 & 0,19 & 1,77 \\
\hline 30 & Myrsine guianensis (Aubl.) Kuntz. & 5 & 15,89 & 2,66 & 0,028 & 0,83 & 0,75 & 0,17 & 1,76 \\
\hline 31 & Bowdichia virgiloides H.B. \& K. & 3 & 9,53 & 2,00 & 0,098 & 0,50 & 0,56 & 0,60 & 1,67 \\
\hline 32 & Myrcya lingua Berg. & 4 & 12,71 & 2,66 & 0,037 & 0,66 & 0,75 & 0,23 & 1,65 \\
\hline 33 & Schefflera macrocarpon (Serm.) D.C. Froolik & 2 & 6,35 & 1,33 & 0,146 & 0,33 & 0,37 & 0,90 & 1,61 \\
\hline 34 & Machaerium sp. & 3 & 9,53 & 2,00 & 0,081 & 0,50 & 0,56 & 0,50 & 1,56 \\
\hline 35 & Hyptidendron canum (Pohl. ex. Benth.) R.M. Harley & ey3 & 9,53 & 2,00 & 0,071 & 0,50 & 0,56 & 0,44 & 1,50 \\
\hline 36 & Roupala montana Aubl. & 3 & 9,53 & 2,00 & 0,036 & 0,50 & 0,56 & 0,22 & 1,28 \\
\hline 37 & Aspidosperma tomentosum A .DC. & 3 & 9,53 & 2,00 & 0,024 & 0,50 & 0,56 & 0,15 & 1,21 \\
\hline 38 & Austroplenckia populnea (Reiss) Lund. & 2 & 6,35 & 1,33 & 0,081 & 0,33 & 0,37 & 0,50 & 1,21 \\
\hline 39 & Tabebuia aurea (Mart.) Bur. & 2 & 6,35 & 1,33 & 0,070 & 0,33 & 0,37 & 0,43 & 1,14 \\
\hline 40 & Tabebuia ochracea (Cham.) Standl. & 2 & 6,35 & 1,33 & 0,042 & 0,33 & 0,37 & 0,26 & 0,97 \\
\hline 41 & Acosmium dasycarpum (Vog.) Yakovl. & 2 & 6,35 & 1,33 & 0,031 & 0,33 & 0,37 & 0,19 & 0,90 \\
\hline 42 & Connarus suberosus Planch. & 2 & 6,35 & 1,33 & 0,028 & 0,33 & 0,37 & 0,17 & 0,88 \\
\hline 43 & Enterolobium gummiferum (Mart.) Macb. & 2 & 6,35 & 1,33 & 0,028 & 0,33 & 0,37 & 0,17 & 0,88 \\
\hline 44 & Vochysia tomentosum Voch. & 2 & 6,35 & 1,33 & 0,013 & 0,33 & 0,37 & 0,08 & 0,79 \\
\hline 45 & Pouteria ramiflora (Mart.) Radlk. & 1 & 3,17 & 0,66 & 0,068 & 0,16 & 0,18 & 0,42 & 0,77 \\
\hline 46 & Myrcia sp. & 2 & 6,35 & 1,33 & 0,010 & 0,33 & 0,37 & 0,06 & 0,77 \\
\hline 47 & Vochysia cinnamomea Pohl. & 1 & 3,17 & 0,66 & 0,060 & 0,16 & 0,18 & 0,37 & 0,72 \\
\hline 48 & Platymenia reticulata Benth. & 1 & 3,17 & 0,66 & 0,048 & 0,16 & 0,18 & 0,30 & 0,65 \\
\hline 49 & Rourea induta Planch. & 1 & 3,17 & 0,66 & 0,029 & 0,16 & 0,18 & 0,17 & 0,53 \\
\hline 50 & Leandra $\mathrm{sp}$ & 1 & 3,17 & 0,66 & 0,027 & 0,16 & 0,18 & 0,17 & 0,52 \\
\hline 51 & Himatanthus obovatus (M. Arg.) R.E. Woodson & 1 & 3,17 & 0,66 & 0,022 & 0,16 & 0,18 & 0,14 & 0,49 \\
\hline 52 & Connarus sp. & 1 & 3,17 & 0,66 & 0,01 & 0,16 & 0,18 & 0,08 & 0,43 \\
\hline 53 & Casearia sylvestris $\mathrm{Sw}$. & 1 & 3,17 & 0,66 & 0,009 & 0,16 & 0,18 & 0,05 & 0,41 \\
\hline 54 & Psidium australe Camb. & 1 & 3,17 & 0,66 & 0,007 & 0,16 & 0,18 & 0,04 & 0,40 \\
\hline 55 & Vochysia tucanorum Mart. & 1 & 3,17 & 0,66 & 0,007 & 0,16 & 0,18 & 0,04 & 0,40 \\
\hline \multirow[t]{2}{*}{56} & Não identificadas & 3 & 9,53 & 2,00 & 0,030 & 0,50 & 0,56 & 0,18 & 1,25 \\
\hline & Total & 600 & 1907 & 354 & 16,253 & 100 & 100 & 100 & 300 \\
\hline
\end{tabular}

Seq. = seqüência das espécies pelo IVI; $\mathrm{N}^{\mathrm{o}}=$ número de indivíduos na amostra; $\mathrm{DA}_{\mathrm{x}}=$ densidade absoluta das plantas por hectare; $\mathrm{DR}_{\mathrm{x}}=$ densidade relativa $(\%) ; \mathrm{FA}_{\mathrm{x}}=$ freqüência absoluta; $\mathrm{FR}_{\mathrm{x}}=$ freqüência relativa $(\%) ; \operatorname{DoA}_{\mathrm{x}}=$ dominância absoluta $\left(\mathrm{m}^{2} / \mathrm{ha}\right) ;$ DoR $_{\mathrm{x}}=$ dominância relativa $(\%)$. As espécies vegetais estão ordenadas pela ordem de importância. 
Tabela 3. Espécies e parâmetros fitossociológicos, ordenados de acordo com os valores do Índice IVI (Índice de Valor de Importância) das espécies na área 2. Parque Estadual da Serra de Caldas Novas (1999).

\begin{tabular}{|c|c|c|c|c|c|c|c|c|c|}
\hline Seq. & Espécies vegetais da Área 2 & $\mathbf{N}$ & $\mathbf{D A}_{\mathbf{x}}$ & $\mathbf{F A} \mathbf{A}_{\mathbf{x}}$ & $\operatorname{DoA}_{x}$ & $\mathbf{D R}_{\mathbf{x}}$ & $\mathbf{F R}_{\mathrm{x}}$ & $\operatorname{DoR}_{x}$ & IVI \\
\hline 1 & Pouteria ramiflora (Mart.) Radlk. & 54 & 191,16 & 30,66 & 1,994 & 9,00 & 8,19 & 10,24 & 27,44 \\
\hline 2 & Qualea parviflora Mart. & 39 & 138,06 & 24,66 & 1,988 & 6,50 & 6,59 & 10,21 & 23,31 \\
\hline 3 & Qualea grandiflora Mart. & 34 & 120,36 & 20,66 & 1,972 & 5,66 & 5,52 & 10,13 & 21,32 \\
\hline 4 & Caryocar brasiliense Camb. & 34 & 120,36 & 20,66 & 1,498 & 5,66 & 5,52 & 7,70 & 18,89 \\
\hline 5 & Vochysia cinamommea Pohl. & 39 & 138,06 & 24,66 & 0,674 & 6,50 & 6,59 & 3,46 & 16,56 \\
\hline 6 & Ouratea hexasperma (St. Hil.) Benth. & 34 & 120,36 & 20,00 & 0,850 & 5,66 & 5,34 & 4,37 & 15,38 \\
\hline 7 & Kielmeyera coriacea (Spreng.) Mart. & 31 & 109,74 & 19,33 & 0,767 & 5,16 & 5,16 & 3,94 & 14,27 \\
\hline 8 & Platimenia reticulata Benth. & 30 & 106,20 & 18,00 & 0,632 & 5,00 & 4,81 & 3,24 & 13,06 \\
\hline 9 & Sclerolobium aureum (Tul.) Benth. & 18 & 63,72 & 10,00 & 1,132 & 3,00 & 2,67 & 5,81 & 11,49 \\
\hline 10 & Palicourea rigida Kunth & 22 & 77,88 & 13,33 & 0,264 & 3,66 & 3,56 & 1,35 & 8,58 \\
\hline 11 & Licania humilis Cham \& Schlect. & 14 & 49,56 & 8,66 & 0,437 & 2,33 & 2,31 & 2,24 & 6,89 \\
\hline 12 & Austroplenckia populnea (Reiss.) Lund. & 14 & 49,56 & 9,33 & 0,378 & 2,33 & 2,49 & 1,94 & 6,77 \\
\hline 13 & Byrsonima verbascifolia Rich. ex Adr. Juss & 16 & 56,64 & 10,00 & 0,278 & 2,66 & 2,67 & 1,42 & 6,76 \\
\hline 14 & Bowdichia virgiloides Kunth. & 8 & 28,32 & 5,33 & 0,740 & 1,33 & 1,42 & 3,80 & 6,56 \\
\hline 15 & Byrsonima coccolobifolia H.B. \& K. & 16 & 56,64 & 10,00 & 0,227 & 2,66 & 2,67 & 1,16 & 6,50 \\
\hline 16 & Brosimum gaudichaudii Tréc. & 7 & 24,78 & 4,66 & 0,651 & 1,16 & 1,24 & 3,34 & 5,75 \\
\hline 17 & Aspidosperma dasicarpon A DC. & 12 & 42,48 & 6,66 & 0,350 & 2,00 & 1,78 & 1,79 & 5,58 \\
\hline 18 & Vochysia tucanorum Mart. & 12 & 42,48 & 8,00 & 0,191 & 2,00 & 2,13 & 0,98 & 5,12 \\
\hline 19 & Qualea multiflora Mart. & 9 & 31,86 & 6,00 & 0,347 & 1,50 & 1,60 & 1,78 & 4,89 \\
\hline 20 & Sclerolobium paniculatum var. subvelutinum Vog. & 6 & 21,24 & 3,33 & 0,542 & 1,00 & 0,89 & 2,78 & 4,67 \\
\hline 21 & Piptocarpha rotundifolia (Less.) Baker & 10 & 35,40 & 6,66 & 0,232 & 1,66 & 1,78 & 1,19 & 4,64 \\
\hline 22 & Pterodon emaginatus Vog. & 6 & 21,24 & 4,00 & 0,38 & 1,00 & 1,06 & 1,98 & 4,05 \\
\hline 23 & Strychnos pseudo-quina St. Hil. & 9 & 31,86 & 6,00 & 0,126 & 1,50 & 1,60 & 0,65 & 3,75 \\
\hline 24 & Hymenia stigonocarpa Mart. ex. Hayne & 7 & 24,78 & 4,66 & 0,248 & 1,16 & 1,24 & 1,27 & 3,68 \\
\hline 25 & Styrax ferrugineus Nees \& Mart. & 8 & 28,32 & 5,33 & 0,116 & 1,33 & 1,42 & 0,60 & 3,36 \\
\hline 26 & Erythroxylum suberosum St. Hil. & 8 & 28,32 & 5,33 & 0,111 & 1,33 & 1,42 & 0,57 & 3,33 \\
\hline 27 & Eremanthus sp. & 8 & 28,32 & 5,33 & 0,109 & 1,33 & 1,42 & 0,56 & 3,32 \\
\hline 28 & Stryphnodendron polyphyllum Mart. var. villosum & 8 & 28,32 & 5,33 & 0,067 & 1,33 & 1,42 & 0,34 & 3,10 \\
\hline 29 & Stryphnodendron adstringens (Mart.) Coville & 7 & 24,78 & 4,00 & 0,168 & 1,16 & 1,06 & 0,86 & 3,10 \\
\hline 30 & Tabebuia aurea (Mart.) Bur. & 7 & 24,78 & 4,66 & 0,124 & 1,16 & 1,24 & 0,64 & 3,05 \\
\hline 31 & Hyptidendron canum (Pohl. Ex. Benth.) R.M. Harley & & 21,24 & 4,00 & 0,191 & 1,00 & 1,06 & 0,98 & 3,05 \\
\hline 32 & Myrcia sp. & 7 & 24,78 & 4,66 & 0,094 & 1,16 & 1,24 & 0,48 & 2,90 \\
\hline 33 & Connarus suberosus Planch. & 5 & 17,70 & 3,33 & 0,148 & 0,83 & 0,89 & 0,76 & 2,48 \\
\hline 34 & Tocoyena formosa K. Schum. & 6 & 21,24 & 4,00 & 0,076 & 1,00 & 1,06 & 0,39 & 2,46 \\
\hline 35 & Aspidosperma macrocarpon Mart. & 5 & 17,70 & 3,33 & 0,129 & 0,83 & 0,89 & 0,66 & 2,39 \\
\hline 36 & Vatairea $\mathrm{sp}$ & 5 & 17,70 & 3,33 & 0,108 & 0,83 & 0,89 & 0,55 & 2,27 \\
\hline 37 & Roupala montana Aubl. & 3 & 10,62 & 2,00 & 0,115 & 0,50 & 0,53 & 0,59 & 1,62 \\
\hline 38 & Tabebuia ochracea (Cham.) Standl. & 4 & 14,16 & 2,66 & 0,034 & 0,66 & 0,71 & 0,17 & 1,55 \\
\hline 39 & Lafoensia pacari St.Hil. & 3 & 10,62 & 2,00 & 0,088 & 0,50 & 0,53 & 0,45 & 1,48 \\
\hline 40 & Annona crassiflora Mart. & 2 & 7,08 & 1,33 & 0,149 & 0,33 & 0,35 & 0,76 & 1,45 \\
\hline 41 & Myrcia lingua Berg. & 3 & 10,62 & 2,00 & 0,036 & 0,50 & 0,53 & 0,18 & 1,22 \\
\hline 42 & Aspidosperma tomentosum Mart. & 2 & 7,08 & 1,33 & 0,091 & 0,33 & 0,35 & 0,46 & 1,15 \\
\hline 43 & Enterolobium gummiferum (Mart.) Macb. & 2 & 7,08 & 1,33 & 0,054 & 0,33 & 0,35 & 0,28 & 0,96 \\
\hline 44 & Hancornia speciosa Nees \& Mart. & 2 & 7,08 & 1,33 & 0,049 & 0,33 & 0,35 & 0,25 & 0,94 \\
\hline 45 & Kielmeyera speciosa St. Hil. & 1 & 3,54 & 0,66 & 0,094 & 0,16 & 0,17 & 0,48 & 0,83 \\
\hline 46 & Erythroxylum tortuosum Mart. & 2 & 7,08 & 1,33 & 0,025 & 0,33 & 0,35 & 0,13 & 0,82 \\
\hline 47 & Diospyros hispida A DC. & 2 & 7,08 & 1,33 & 0,013 & 0,33 & 0,35 & 0,07 & 0,76 \\
\hline 48 & Himathantus obovatus (M.Arg.) R.E. Woodson & 2 & 7,08 & 1,33 & 0,011 & 0,33 & 0,35 & 0,05 & 0,74 \\
\hline 49 & Peltogyne sp. & 1 & 3,54 & 0,66 & 0,049 & 0,16 & 0,17 & 0,25 & 0,60 \\
\hline 50 & Salvertia convallariaeodora St. Hil. & 1 & 3,54 & 0,66 & 0,049 & 0,16 & 0,17 & 0,25 & 0,60 \\
\hline 51 & Acosmium dasycarpum (Vog.) Yakovl. & 1 & 3,54 & 0,66 & 0,023 & 0,16 & 0,17 & 0,12 & 0,46 \\
\hline 52 & Dalbergia miscolobium Benth. & 1 & 3,54 & 0,66 & 0,019 & 0,16 & 0,17 & 0,09 & 0,44 \\
\hline 53 & Machaerium sp. & 1 & 3,54 & 0,66 & 0,019 & 0,16 & 0,17 & 0,09 & 0,44 \\
\hline 54 & Neea theifera Oerst. & 1 & 3,54 & 0,66 & 0,019 & 0,16 & 0,17 & 0,09 & 0,44 \\
\hline 55 & Leandra $\mathrm{sp}$. & 1 & 3,54 & 0,66 & 0,012 & 0,16 & 0,17 & 0,06 & 0,40 \\
\hline 56 & Andira humilis Mart. ex Benth. & 1 & 3,54 & 0,66 & 0,011 & 0,16 & 0,17 & 0,05 & 0,40 \\
\hline 57 & Casearia sylvestris Sw. & 1 & 3,54 & 0,66 & 0,007 & 0,16 & 0,17 & 0,03 & 0,38 \\
\hline 58 & Miconia sp. & 1 & 3,54 & 0,66 & 0,004 & 0,16 & 0,17 & 0,02 & 0,36 \\
\hline \multirow[t]{2}{*}{59} & Não identificadas & 1 & 3,54 & 0,66 & 0,119 & 0,16 & 0,17 & 0,61 & 0,95 \\
\hline & Total & 60 & 2124 & 374 & 19,462 & 100 & 100 & 100 & 300 \\
\hline
\end{tabular}

Legenda - Idem a tabela 2. 
amplamente distribuída, estando presente tanto em áreas de cerrado sensu stricto, cerradão e até mesmo em campo cerrado (embora com densidades mais baixas).

A densidade total estimada para a área 1 foi de 1907 plantas ha ${ }^{-1}$ e para a área 2 foi de 2124 plantas $\mathrm{ha}^{-1}$. Esses valores aproximaramse dos que foram observados por Goodland (1979) que, incluindo plantas que tivessem no mínimo $10 \mathrm{~cm}$ de diâmetro de caule no nível do solo, encontrou 2253 plantas ha ${ }^{-1}$. Oliveira et al. (1982) e Ribeiro (1983) registraram 600 plantas ha ${ }^{-1}$, mas o método de inclusão destes autores foi de $10 \mathrm{~cm}$ de diâmetro do caule à altura do peito (DAP), enquanto Medeiros (1983) e Ribeiro et al. (1985) encontraram valores próximos a 1000 plantas ha $^{-1}$, sendo que no primeiro trabalho foram incluídas plantas com no mínimo $10 \mathrm{~cm}$ de DAP e no segundo foram incluídas aquelas que tivessem $3 \mathrm{~cm}$ de DAP e/ou $2 \mathrm{~m}$ de altura de fuste. Todos os trabalhos citados foram feitos em cerrado sensu-stricto. No cerradão, Ribeiro (1983) e Ribeiro et al. (1985) obtiveram, respectivamente, 1864 e 2231 plantas ha' ${ }^{-1}$, usando os critérios de inclusão já comentados. Esses valores aproximam-se dos resultados obtidos na Serra de Caldas Novas em área de cerrado sensu stricto mas a comparação torna-se difícil, pois os critérios de inclusão foram diferentes.

Os valores dos vários parâmetros fitossociológicos encontram-se nas (Tab. 2 e 3), onde as populações foram ordenadas por ordem decrescente de IVI. A seqüência decrescente de espécies em função do IVI nas áreas amostradas pode ser observada na Fig. 1.

As dez espécies de maior IVI na área 1 foram: Kielmeyera coriacea, Qualea grandiflora, Caryocar brasiliense, Syagrus flexuosa, Ouratea hexasperma, Qualea multiflora, Qualea parviflora, Annona crassiflora, Eremanthus sp. e Byrsonima verbascifolia, totalizando $60,05 \%$ do IVI total. $\mathrm{Na}$ área 2, as espécies de maior IVI foram: Pouteria ramiflora, Qualea parviflora, Qualea grandiflora, Caryocar brasiliense, Vochysia cinamommea, Ouratea hexasperma, Kielmeyera coriacea, Platimenia reticulata, Sclerolobium aureum e Palicourea rigida, representando 56,78\% do IVI total. Dessas, apenas $K$. coriacea, Q. grandiflora, $C$. brasiliense, $O$. hexasperma e Q. parviflora estiveram entre os dez maiores IVIs nas duas áreas (Tab. 2 e 3). Na área 1, K. coriacea apresentou o maior IVI, principalmente devido à sua densidade e frequiência, sendo que sua dominância foi menor que as de $Q$. grandiflora e $C$. brasiliense. Já $S$. flexuosa, palmeira muito difundida em áreas de cerrado e mata (Almeida et al., 1998) teve frequiência maior que $Q$. grandiflora e $C$. brasilien$s e$, entretanto sua dominância foi baixa, menor que a de $O$. hexasperma, $Q$. multiflora e $A$. crassiflora que ocuparam posições abaixo da sua. Eremanthus sp apresentou frequiência e densidade maiores que $A$. crassiflora mas a sua dominância foi baixa, colocando-a em nona posição, enquanto $A$. crassiflora ocupou a oitava posição.

$\mathrm{Na}$ área 2, $P$. ramiflora foi a espécie que apresentou maior IVI e também os maiores valores nos três parâmetros (densidade, frequiência e dominância). V. cinamommea (a $5^{\mathrm{a}} \mathrm{em}$ IVI), apesar de apresentar maior densidade e freqüência que C. brasiliense ( $\left.4^{\mathrm{a}}\right)$ e $Q$. grandiflora $\left(3^{\mathrm{a}}\right)$, teve baixa dominância, porque seus caules não são muito espessos. Comportamento oposto foi exibido por Brosimum gaudichaudii (16 em IVI), cuja dominância foi maior que Platimenia reticulata, Palicourea rigida, Licania humilis, B. verbascifolia, Austroplenckia populnea e B. coccolobifolia, todas com IVI mais alto que o seu. Outras espécies como: Aspidosperma dasicarpon, V. tucanorum, Q. multiflora, Piptocarpha rotundifolia, Strychnos pseudo-quina, Styrax ferrugineus, Erytroxylum suberosum, Eremanthus sp. e Stryphnodendron polyphyllum apresentaram densidade maior que $B$. gaudichaudii mas ocuparam posições inferiores em importância, pois apresentaram dominâncias mais baixas. O coeficiente de similaridade de Jaccard entre as duas áreas foi de 0,72 e o índice de Morisita apresentou um valor um pouco menor, 

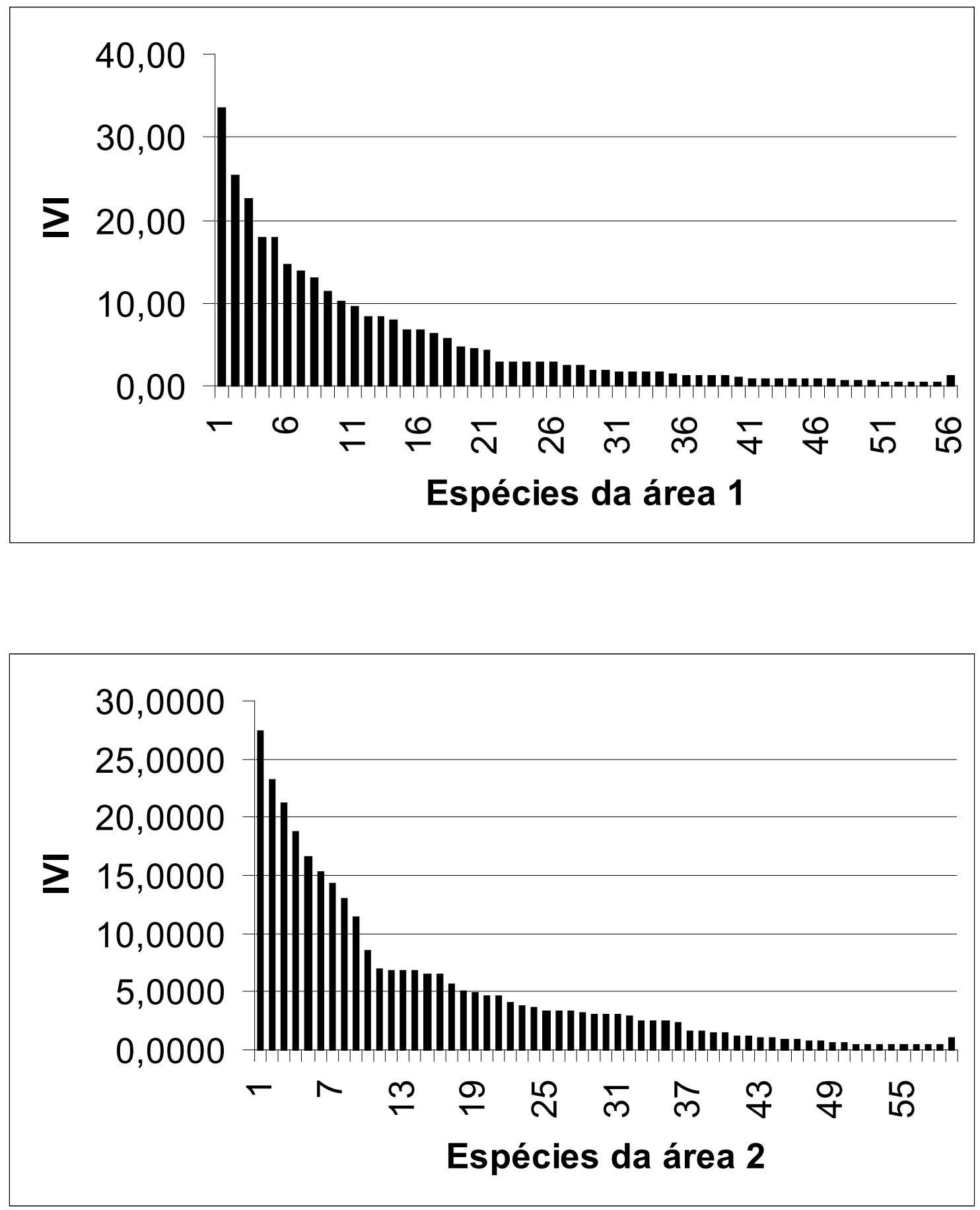

Figura 1. Seqüência decrescente das populações das áreas 1 (A1) e 2 (A2) em função do índice de valor de importância (IVI). Os números correspondem às espécies de acordo com as tabelas 1 e 2, respectivamente. Parque Estadual da Serra de Caldas Novas, GO. 
0,64. Esses dois valores indicam que a flora das áreas amostradas possui grande similaridade, tanto do ponto de vista da composição de espécies quanto dos padrões de abundância de suas populações. A Tab. 4 mostra uma comparação dos Coeficientes de Jaccard (Krebs, 1989) entre os levantamentos fitossociológicos de Ribeiro et al. (1985) em Planaltina - DF, Nascimento \& Saddi (1992) em Cuiabá - MT, Meireles \& Barreto Luiz (1995) em Brasília - DF e Manoel (1999) na Ser- ra Dourada - GO, com as áreas estudadas na serra de Caldas Novas. Observou-se que, de modo geral, as similaridades foram baixas, mostrando que o cerrado apresenta uma grande heterogeneidade.

As médias e desvios padrões das medidas de altura das plantas e circunferências dos caules nas duas áreas foram: Altura das plantas (área $1=$ $252,38 \pm 110,20$ e área $2=304,15 \pm 141,22)$ e as circunferências dos caules (área $1=29,16 \pm 14,83$ e área $2=30,00 \pm 14,86$ ) (Fig. 2).

Figura 2. Médias de altura e circunferência nas áreas 1 (A1) e 2 (A2) analisadas no Parque Estadual da Serra de Caldas Novas. Variação da altura $(\mathrm{cm})(\mathrm{t}=7.078, \mathrm{p}<0.001)$ e de circunferência $(\mathrm{cm})(\mathrm{t}=1.361, \mathrm{p}=0.174)$.

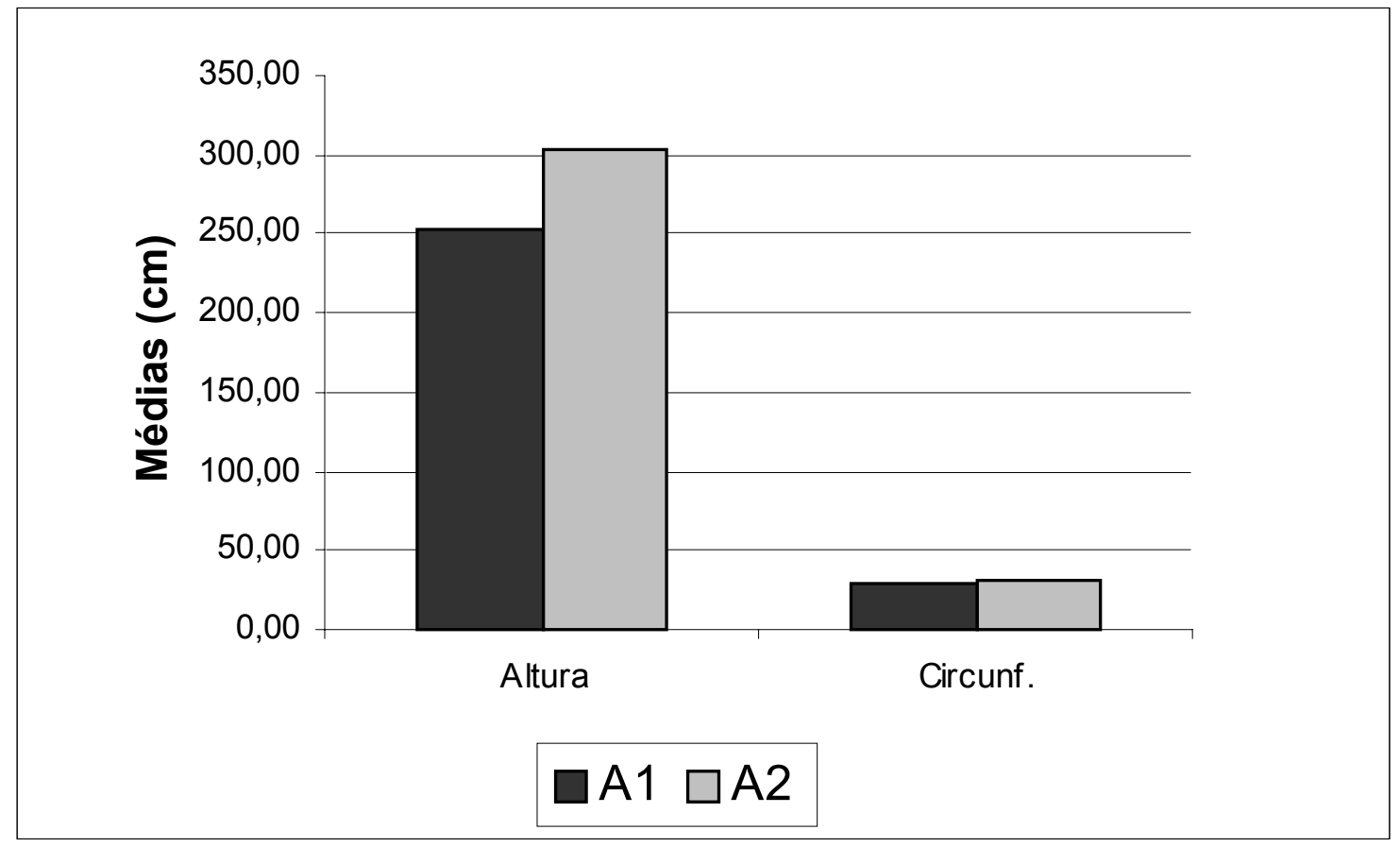

Tabela 4. Coeficiente de Jaccard entre as comunidades vegetais encontradas nos trabalhos de alguns autores e nas áreas estudadas na Serra de Caldas Novas.

\section{Área 1 Área 2}

Ribeiro et al. (1985) Planaltina - DF

$0,31 \quad 0,33$

Nascimento \& Saddi (1992) Cuiabá - MT

$0,16 \quad 0,18$

Meireles \& Barreto Luiz (1995) Brasília - DF

$0,22 \quad 0,24$

Manoel (1999) Serra Dourada - Mossâmedes - GO

0,21

0,19 
O teste $\mathrm{t}(7.078, \mathrm{p}<0.001)$ mostrou que as duas áreas apresentaram diferenças significativas em alturas das árvores, mas em relação às circunferências, o teste $\mathrm{t}(1.361$, $\mathrm{p}=0.174)$ demonstrou que elas não diferem significativamente. As áreas analisadas no Parque Estadual da Serra de Caldas Novas são bem similares, mas apesar disso a ocorrência de algumas espécies foi muito diferente de uma área para outra. Syagrus flexuosa e Annona crassiflora, apresentaram grande importância na área 1 e quase não foram encontradas na área 2. Por outro lado, Pouteria ramiflora, Vochysia cinamommea e Platimenia reticulata destacaram-se na área 2 e quase não foram observadas na área 1. É possível que as variações minerais ou até mesmo a drenagem do solo, influenciem na distribuição dessas populações no Parque. A área 1 apresenta um latossolo vermelho escuro e a área 2 apresenta um latossolo variando de vermelho escuro para o vermelho amarelo. Estes tipos de solos possuem mecanismos de drenagem de água ligeiramente diferenciados.

O latossolo vermelho-amarelo drena a água mais lentamente que o vermelho escuro (Reatto et al., 1998). Dessa maneira, as plantas da área 2 teriam água disponível por mais tempo que as da área 1, o que poderia explicar as diferenças na distribuição das populações.

\section{Agradecimentos}

Os autores agradecem à Agência Goiana de Meio Ambiente e Recursos Naturais pela concessão da licença de pesquisa, aos funcionários do PESCAN pela atenção dispensada à equipe, e especialmente à Sra. Magali Izuawa, diretora da unidade, cujo apoio foi fundamental para viabilizar o trabalho.

\section{Referências Bibliográficas}

Almeida, A . F. \& Sarmento, F. N. M. (coord.). s.d. Parque Estadual da Serra de Caldas - Plano de Manejo. CTE (Centro Tecnológico de Engenharia Ltda), FEMAGO - Fundação Estadual do Meio Ambiente, Goiânia.

Almeida, S.P.; Proença, C.E.B; Sano, S. M \& Ribeiro, J.F. 1998. Cerrado: espécies vegetais úteis. Planaltina: EMBRAPA-CPAC - DF. 464p.

Castro, A.A.J.F. 1994. Comparação Florística de Espécies do Cerrado. Silvicultura. 15(58):16-18.

Eiten, G. 1993. Vegetação do Cerrado. In: Novaes Pinto, $\mathrm{M}$ (org.). Cerrado: caracterização, ocupação e perspectivas. Brasília. Editora Universidade de Brasília.. 17 - 73.

Felfili, J.M. 1994. Floristic composition and phytosociology of the gallery forest alongside the Gama Strem in Brasília, DF, Brazil. Revista Brasileira de Botânica. 17(1):1-11.

Felfili, J. M. \& Silva Jr, M. C. 1992. Floristic composition, phytosociology and comparison of cerrado and gallery forests at Fazenda Água Limpa, Federal District, Brazil. In: Nature and dynamics of forest-savanna boundaries. (P.A ., Furley, J.A ., Proctor and J. A ., Ratter eds.) Chapman \& Hall, London. 393-415

Felfili, J. M. \& Silva Jr, M. C. 1993. A comparative study of cerrado (sensu stricto) vegetation in Central Brazil. Journal Tropical of Ecology. 9: 277289.

Felfili, J. M.; Silva Jr, Rezende, M. C.A; Machado, V. J. W. B; Walter, B. M. T; Silva, P. E. N \& Hay, J.D. 1993. Análise comparativa da florística e fitossociologia da vegetação arbórea do cerrado sensu stricto na Chapada Pratinha, DF - Brasil. Acta Botanica Brasilica. 6(2):27-46.

Filgueiras, T.S. \& Pereira, B. A S. 1993. Flora do Distrito Federal. In: Novaes Pinto, M (org.). Cerrado: caracterização, ocupação e perspectivas. Brasília. Editora Universidade de Brasília. 345-404.

Goodland, R.J. A . 1970. Plants of the cerrado vegetation of Brazil. Phytologia. 20:57-77.

Goodland, R. 1979. Análise ecológica da Vegetação do Cerrado. In Ecologia do Cerrado. (R. Goodland \& M. G. Ferri, eds.). Itatiaia. Belo Horizonte. 61-171.

Krebs, C. J. 1989. Ecological Methodology. Harper and Row. New York. USA.

Manoel, L. C. 1999. Composição florística, fitossociologia e estado nutricional de comunidades arbóreas de um cerrado rupestre e um cer- 
rado ralo na Serra Dourada - Goiás. Dissertação de Mestrado. Universidade Federal de Goiás.

Mantovani, W. \& Martins, F. R. 1993. Florística do Cerrado na Reserva Biológica de Moji Guaçu, SP. Acta Botanica Brasilica. 7(1):33-60.

Medeiros, R.A . 1983. Comparação de algumas espécies acumuladoras e não acumuladoras de alumínio nativas do cerrado. Dissertação de Mestrado. UnB, Brasília. 94 p.

Meirelles, M. L. \& Barreto Luiz, A .J. 1995. Padrões espaciais de árvores de um cerrado em Brasília, DF. Revista Brasileira de Botânica. 18(2)185-189.

Mitermeier, N.; Myers, R.A. \& Mittermeier, C.G.1999. HOTSPOTS - Earth's Biologically Richest and Most Endangered Terrestrial Ecoregions. CEMEX - Conservation International. Mexico City. 430p.

Müeller-Dombois, D \& Ellemberg, H. 1974. Aims and methods of vegetation ecology, New York, John Willey \& Sons, 547p.

Nascimento, M. T. \& Saddi, N. 1992. Structure and florist in composition in an area of cerrado in Cuiabá - MT, Brazil. Revista Brasileira de Botânica. 15(1):47-55.

Oliveira, P.E.A .M.; Pereira, L.A; Lima, V. L.G.F.; Franco, A .C.; Barbosa, A . A.; Batmanian, G. J. \& Moura, L. C. 1982. Levantamento preliminar de um Cerrado no Parque Nacional de Brasília. Boletim Técnico do IBDF. 7:25-31.

Oliveira-Filho, A . T. \& Martins, F. R. 1986. Distribuição, caracterização e composição florística das formações vegetais da região da Salgadeira, na Chapada dos Guimarães (MT). Revista Brasileira de Botânica. 9:207-223

Oliveira-Filho, A . T.; Shepherd, G. J.; Martins, F. R.\& Stubblebine, W. H. 1989. Environmental factors affecting physiognomic and floristic variation in an area of cerrado in central Brazil. Journal of Tropical Ecology. 5:413-431.

Pedralli, G.; Freitas, V. L. O.; Meyer, S. T.; Teixeira,
M.C.B. \& Gonçalves A .P.S. 1997. Levantamento florístico na Estação ecológica do Tripuí, Ouro Preto, MG. Acta Botanica Brasilica 11(2): 191-213.

Ratter, J. A . 1986. Notas sobre a vegetação da Fazenda Água Limpa (Brasília, DF, Brasil). Editora UnB. Textos Universitários n. 003. Brasília.

Ratter, J. A.; Richards, P. W.; Argent G. \& Gifford D. R.. 1973. Observations on the vegetation of northeastern Mato Grosso. The woody vegetation types of the Xavantina-Cachimbo expedition area. Phil. Trans. R. Soc. 226(B):449-492

Ratter, J.A . \& Dargie, T.C.D. 1992. An analysis of the floristic composition of 26 cerrado areas in Brazil. Edinburg Journal of Botany. 49(2):235-250.

Ratter, J.A; Bridgewater, S.; Atkinson, R.; Ribeiro, J.F. 1996. Analysis of the floristic composition of the Brazilian Cerrado vegetation II: comparison of the woody vegetation of 98 areas. Edinburg Journal of Botany. 53(2):153-180

Reatto, A; Correia J. R. \& Spera, S. T. 1998. Solos do Bioma Cerrado: aspectos pedológicos. In: SANO, S. M. \& Almeida, S. P. Cerrado - ambiente e flora. $47-86$.

Ribeiro, J.F. 1983. Comparação da concentração de nutrientes na vegetação arbórea e nos solos de um cerrado e um cerradão no Distrito Federal, Brasil. Dissertação de Mestrado. Universidade de Brasília, Brasília - DF.

Ribeiro, J. F.; Silva, J. C. S. \& Batmanian, G. J.. 1985. Fitossociologia de tipos fisionômicos de cerrado em Planaltina - DF. Revista Brasileira de Botânica. 8:131-142.

Rizzo, J. A .1981. Flora do Estado de Goiás: Coleção Rizzo. Ed. Universidade Federal de Goiás. Goiânia - GO. 35 p.

Silberbauer-Gottsberger, I. \& Eiten, G. 1983. Fitossociologia de um hectare de cerrado. Brasil Florestal. 54:55-70.

Sokal, R. R. \& Rolhf, F.J. 1995. Biometry. $3^{\mathrm{a}}$ ed. New York: W. H. Freeman and Company. 
